\title{
LOS TIPOS MONETARIOS RELACIONADOS CON LA DOMUS IMPERIAL EN ADRIANO Y ANTONINO PÍO
}

\author{
JOSÉ ANTONIO GARZÓN BLANCO
}

\begin{abstract}
En las múltiples acuñaciones monetarias de la Roma Imperial existen cierto número de representaciones que tienen más vinculación que otras con el palacio de los emperadores. Son aquéllas que reflejan los actos de la vida cotidiana y más especialmente, la ideología imperial en relación con su propia familia y con el ámbito que les rodea. Estando dirigidas estas emisiones, al restringido radio de Roma y sus alrededores, o como mucho, a la provincia de Italia. Vamos a referirnos a algunos de estos tipos o mensajes monetarios, que muchas veces, transmiten una doble idea: Providentia - Adoptio. Roma - Romulus y Honos - Iuventas, y de una forma más general, Libertas Pvblica.

Dans cet article on analyse la propagande numismatique d'Hadrien, d'Antonin le Pieux, et de Marc-Aurele avec la fonction de prince, tout en relation avec les emissions monétaires de la maison imperiale.

Hadrien suppose le point le plus grand, et le plus interessant en relation à la propagande imperiale dans la numismatique. En effect, l'empereur raconte la totalité de son gouvernament dans les monnaies, en plus de sa politique.

Pource qui respecte a Antonin le Pieux, sa monnaie est la continuation de celle d'Hadrien, dans la chronologie et dans leur intention, mais la divulgation de leurs idées dans les monnayages est beaucoup plus prolixe et plus confuse.
\end{abstract}

UN ASPECTO ESPECIAL

\section{DE LA PROVIDENCIA. LA ADOPTIO}

La adoptio es la característica política más importante del siglo II, Trajano fue adoptado por Nerva, Adriano por Trajano, Antonino Pio por Adriano y Marco Aurelio y Lucio Vero por Antonino Pio.

Trajano conmemora en la moneda su adoptio a través de la simbólica entrega del globo terráqueo por parte de Nerva a su sucesor, tan representativa imagen se repetirá posteriormente en la de Adriano, Trajano utilizará para esta moneda la leyenda PROVID., insinuando con ella la Providentia Deorum, que interviene para que a través del buen go- bierno de Trajano, Roma y el Imperio alcancen la máxima felicitas (1).

Según Mazzarino (MAZZARINO, 1980, 316317), «la adoptio es la forma constitucional que toman los Antoninos, y antes que ellos Nerva, para

(1) MATTINGLY y SYDENHAM, (1926, II, n. $\left.{ }^{\circ} 28\right)$; COHEN, (1880-1892, II, nos. 318-319). Una gran cantidad de obras tratan el tema de la adoptio de Adriano, fundamentalmente: BERANGER, (1939, 171-187); CAMUZZI, (1900, 157 ss); CARCOPINO, (1949, 262 ss); CARCOPINO, (1965, 67-69); DEN BOER, (1955, 277-286); FLERES, (1964, 199 205); GEER, (1936, 47-54); GRENADE, (1950, 258 SS); MERTEN, (1977, 247). 
asegurar la sucesión dinástica. Para esto se basaron en la tradición y en un ideal romano: la monarquía «adoptiva» sería sólo un reflejo, desde el punto de vista ideológico, de la repugnancia romana a la pura concesión dinástica. Ello tiene un buen reflejo en el discurso de Galba adoptando a Pisón, en el primer libro de las Historiae de Tácito se manifiesta la ideología romana, por la cual el Imperio no debe considerarse como unius familiae hereditas, sino que por el contrario, debe encomendar al emperador la nobilísima y delicadísima misión de escoger al optimus, a través de la adoptio. Nerva, que no había tenido hijos, adoptó a Trajano, y éste en sus últimos momentos, ya cerca de la muerte, adoptó a su vez a Adriano. Y parece que esta elección no se hizo tanto por la voluntad de Trajano, sino por la de su esposa Plotina. Esta tardía y desinteresada designación da la impresión de una atormentada incertidumbre, de una notable indecisión de Trajano, lo que no parece indicar una excesiva deferencia hacia Adriano. Trajano habría intuido que Adriano había de dar un nuevo rumbo a la política del Imperio».

La adoptio se realizó el día quinto antes de las idus de agosto, el 9 de agosto del año 117, cuando Adriano era legatus de Siria, dos días después le llegaron las noticias de la muerte del emperador; a partir de ese momento, Adriano celebró su dies imperii el 11 de agosto, (2).

«Trajano no había tenido hijos en su matrimonio. Pero su esposa Plotina y el prefecto de los pretorianos, Acilius Attianus, aseguraron que en su lecho de muerte el difunto había adoptado a su pariente varón más próximo, Publius Elius Hadrianus, de 41 años de edad. Sobre la base de esa indicación, el ejército del Este le proclamó emperador. Que la adoptio fuera efectivamente formulada, fue puesta en tela de juicio desde el principio, como hace en los Annales el propio Tácito, cuando habla de los deseos de Livia para conseguir el trono para Tiberio, es muy probable que en realidad se esté refiriendo a Plotina. No obstante, parece difícil que con el brillante cursus honorum y la protección que le dispensaba Trajano y Attianus se hubiera podido elegir a otra persona», (ALTHEIM, 1955, 101). Por

(2) CIL., VI, $33885=7214$. Cfr. S.H.A., (Hadr. IV, 7); PIR 2 , I, 30, a 184 . tanto, es poco probable que el testamento de Trajano fuese falsificado y los rumores carecerían de fundamento. Adriano, hombre práctico, les dejó decir, mientras se apresuraba a tomar el poder.

En la numismática la adoptio de Adriano queda ampliamente reflejada; así aparece un tipo inequívoco con Trajano y Adriano, cara a cara, dándose la mano derecha en actitud de concordia. Un segundo tipo muestra al emperador de frente a una segunda figura togada, una recibe un globo del otro o le ayuda a soportarlo. Este tipo tenía ya una larga tradición, pues está asociado a la Providentia y ésta puede estar asociada con el emperador precedente o con el Senado, dependiendo del sentido de la moneda. El tipo se presta a dos interpretaciones: si el globo lo soportan ambas figuras, hemos de creer que se trata de los dos emperadores; mientras que si lo lleva una sola de las figuras, se entiende que se trata del «emperador y el Senado». Strack, ve este segundo tipo como una Providentia Senatus, Adriano y el Senado sostendrían el mundo entre ambos. Se basa para ello en que la aparición de los dos emperadores sería una alusión demasiado clara y directa, siendo además, que Adriano buscó la aprobación del Senado para todos su actos. Mattingly sostiene lo contrario, que ambos son emperadores, para lo cual se basa en que sostiene rollos, que es un atributo del emperador, y no llevan el cetro, que es un emblema del Senado. Además, el pose de las dos figuras es el característico de la adoptio. Por lo cual, llega a conclusión de que se trata de una Providentia Augusti, es decir, la Providentia de Trajano a través de la transferencia de poder y de la adoptio de Adriano (MATTINGLY, 1936, III, CXXV).

En una ceca de Oriente, aún mal estudiadas por lo incompleto del material y lo incierto de su atribución, aparece el tipo de la adoptio con todos los títulos de Adriano, lo que vendría a demostrar la intervención del propio emperador en su deseo de dar a conocer su reconocimiento como cabeza del Imperio. En el reverso de esta moneda lleva la leyenda: TRIBVNIC. POTESTAS ADOPTIO, indica el conferimiento de la Tribunicia Potestad de esta acuñación. Es más que probable la existencia de divergencias en el primer año de gobierno ente Adriano y el Senado. Es relevante que el Senado no acuñe monedas con el reverso Adoptio, pero es fácil pensar que los sestertius con Trajano y Adriano en pie, frente a frente, sosteniendo un globo, sustituye perfectamente la falta de una leyenda alusoria, (3). 
No obstante Adriano se declara formalmente hijo de Trajano y nieto de Nerva, en ningún momento va a honrar la memoria de su padre natural, rompiendo la tradición instituida por Trajano; y dejando bien claro que es emperador por derecho propio y para ello va a poner todo su énfasis en las emisiones con Adoptio y Providentia, cuya íntima relación la podemos entender claramente como Providentia Deorum (MATTINGLY, 1936, III, CXXVII).

Además de la Providentia en relación con la adoptio, existen otros tipos cuyo estudio más generalizado se puede hacer a través de dos obras sobre el tema de la Providentia, fundamentalmente el trabajo de M.R. Alfoldi (ALFOLDI, 1955-1956, 245260 ), que estudia las variaciones y el origen de la leyenda, así como la evolución de la idea de Providentia durante el Imperio, y en el mismo sentido el trabajo de R.T. Scott (SCOTT, 1982, 436-439), que aporta interesantes noticias sobre el tema, tanto mitológico como numismático de esta diosa durante el Imperio. Para concretar, además de las monedas acuñadas con la leyenda o con la idea de la adoptio, otras muchas nonedas nos hablan de la Providentia en otros planos distintos. Así, los denarius de los años 119-122, nos hablan de la Providentia, la cual está de pie a la izquierda portando cetro y señalando con la mano derecha con un globo a sus pies. La leyenda del reverso es: P.M.TR.P.COS.III. PRO.AVG. Según Mattingly (4), esta moneda representa la sabia Providentia del emperador, volcado en todos los problemas del gobierno. Dion Cassio recuerda que Adriano quiere expiar serias faltas (DIO. CASS., Hist., LXIL,5). En el año 121 un dupondius muestra la leyenda PROVIDENTIA DEORUM S.C., lo cual expresaría con evidente lenguaje el sagrado derecho de Adriano hacia el trono. Él es el emperador por orden de la divina Providentia, simbolizada por el cetro de Júpiter, que se lo ha enviado desde los cielos y por la propia águila del dios. La adoptio de Adriano no se niega, pero se dice ahora que la adoptio imperial está basada en algo mucho más profundo. En la moneda de Adriano está de pie a la izquierda, recibiendo un ce-

(3) MATTINGLY, (1923, III, CLXII); MATTINGLY y SYDENHAM, (1926, II, nos. 534 a, -535 b).

(4) MATTINGLY, (1936, III, CXXXI); COHEN, (1885-1892, II, $\mathrm{n}^{\circ}$ 1198-2); MATTINGLY y SYDENHAM, (1926, II, $\left.\mathrm{n}^{\circ} 133-\mathrm{C}\right)$. tro que le hace entrega un águila en vuelo desde la derecha (5).

Es muy tardía la siguiente aparición de la iconografía de la alegoría, que no tiene lugar hasta el período de los años 134-138, cuando Adriano celebra sus victorias sobre los judíos, este último tipo lleva la leyenda PROVIDENTIA AVG, y expresa tipos de Vota Suscepta y Vota Pública y la adoptio de Elio (6). Este mismo motivo se repite en la moneda de bronce, de la misma manera la Providentia $A u g .$, nos habla de la sabia precisión del emperador adoptando a su sucesor (MATTINGLY, 1936, III, CLXXXII).

Por último, nuestra principal fuente, la Historia Augusta, nos da amplias referencias cómo se produjo la aceptación de Adriano como hijo adoptivo: Quintum iduum Augustarum diem legatus Syriae litteras adoptionis accepit; Quando et natalem adoptionis celebrari iussit. Tertium iduum earumdem, quando et natalem imperii statuit celebrandum, excessus ei Traiani nuntiatus est (S.H.A., Hadr., IV6).

La adoptio de Elio, aunque carece de reflejo numismático, sí lo tiene en las fuentes: Tunc Ceionium Commodum, Nigrini generum insidiatoris quodam, sibi forma commendatum adoptare constituit. Adoptavit ergo Ceoinium Commodum Verum invitis omnibus eumque Helium Verum Caesarem appellavit (S.H.A., Hadr., XXIII, 10-12). Y más adelante: Ceionius Commodus, qui et Helius Verus appellatus est, quem sibi Hadrianus aevo ingraviscente morbis tristioribus pressus peragrato iam orbe terrarum adoptavit, nihil habet in sua vita memorabile, nisi quod primus tantum Caesar est appellatus, non testamento, ut antea solebat, neque eo modo quo Traianus est adoptatus (S.H.A., Ael., II, 2-3). Hay que hacer, no obstante, una excepción a este reflejo numismático, en una moneda estudiada por H.A. Cahn (CAHN, 1936, 57-58), de acuñación provincial, concretamente de StratonikeiaAdrianopolis, y mandada fabricar por orden del estratega Kandidos para celebrar la adoptio de Elio.

(5) COHEN, (1880-1892, II, nos. 1208-1216); MATTINGLY y SYDENHAM, (1926, II, $n^{\circ} 602$ a-S).

(6) MATTINGLY, (1923, III, CXLV); COHEN, (1880-1892, II, nos. 1201-1202); MATTINGLY y SYDENHAM, (1926, II, $\mathrm{n}^{\circ}$ 262-C). 
Los tipos de Providentia de Antonino son escasos; los primeros de ellos datan de sestertius de la acuñación comprendida entre el 140 al 144, la inscripción es PROVIDENTIA DEORUM S.C., y muestra en el campo, el Rayo Alado, el Divino emblema de Júpiter, el padre de los dioses, es evidente una invocación, dentro del más puro estilo propagandístico, a la Divina Providentia, ordenando debidamente la sucesión (7). Hay que ver en este sentido la obra de M.P. Charleswort (CHARLESWORT, 1936, 107ss), que aunque anticuada, hace un profundo estudio de Providentia, dándole un valor exagerado en relación a otras virtudes imperiales, ya que coloca a Providentia por encima de todas las demás, y esto es inexacto, en la medida que su principal valor es en relación a la adoptio, como hemos visto. La acuñación se continúa durante los años 145 al 147, Mattingly (MATTINGLY, 1940, IV, LXIV), la califica como «el rayo irresistible que el emperador podría llegar a manejar. Es el que se muestra aquí, como símbolo de Júpiter tendido en su "pulvinar". Providentia es emitida inmediatamente después por Marco Aurelio en los años 148 al 149 (MATTINGLY, 1940, IV, LXVII). La última vez que se muestra el rayo alado de Júpiter y la leyenda que hace referencia a la diosa es en sestertius de los años 155 al 156 (MATTINGLY, 1940, IV, $\mathrm{XC}$ ), sin que podamos dar más explicaciones sobre el mismo. Solamente una vez se muestra la personificación de Providentia, pero en un denarius que lleva la leyenda Aeternitas, la diosa en pie a la izquierda con globo y centro (8).

Finalmente, un último tipo de Providentia, en denarius, pertenece a la Diva Faustina, fechado en el año 141, con la leyenda PROV. DEOR. TR.P.COS.II, que muestra a la diosa de pie con globo y cornucopia (MATTINGLY y SYDENHAM, 1930, III, $\left.\mathrm{n}^{\circ} 410-\mathrm{S}\right)$. Es, sin duda, una invocación a la Divina Providencia para que acoja el alma de la difunta.

\section{HONOS}

Una personificación predominantemente militar, está relacionada con Pax y con Virtus, con la primera porque es portadora del ramo de la $\mathrm{Pax}$, con

(7) COHEN, (1880-1892, III, nos. 682-684); MATTINGLY y SYDENHAM, (1930, III, n 618-C).

(8) COHEN, (1880-1892, III, n ${ }^{\circ}$ 20-C); MATTINGLY y SYDENHAM, (1930, III, $\left.\mathrm{n}^{\circ} 114-\mathrm{S}\right)$.
Virtus en su sentido de fuerza y valor. Es por tanto, una personificación predominantemente masculina. Honos se muestra muy pocas veces en la moneda de Antonino Pio, y cuando esto sucede, es para alabar al príncipe, es decir, a Marco Aurelio, y casi siempre està en compañía de Virtus, de tal manera que personifican al príncipe y al emperador, Virtus sería el emblema de Antonino y Honos el de Marco Aurelio. Por ota parte, Honos y Virtus son virtudes eminentemente militares, pero ya en la primera emisión de Antonino con Honos, aproximadamente del año 144, en sestertius con la leyenda en el reverso HONORI AVG. COS. IIII S.C., muestra a Honos de pie y de frente, con la cabeza vuelta a la izquierda, togado sosteniendo el ramo de la paz (en contraposición a su carácter típicamente militar) y cornucopia, Mattingly piensa que habría que investigar la ocasión especial que dio lugar a esta acuñación (MATTINGLY, 1940, IV, LXXXVI). El mismo autor dice que «desde el punto de vista tipológico, Honos y Virtus son la casi exacta copia de la divina pareja: Apolo y Diana» (MATTINGLY, 1940, IV, LVIII). Marco Aurelio comienza casi de inmediato las acuñaciones con Honos en compañía de Hilaritas dos emisiones que en la moneda de Marco van siempre juntas (MATTINGLY; 1940, IV, LXXXV, LXIV). Las siguientes acuñaciones pertenecen en su casi totalidad a Marco, así las que van entre el 145 y el 147 (MATTINGLY, 1940, IV, LXV). De estos últimos tipos dice Mattingly: «Honos es representada en una pose inusual, la cornucopia es análoga a la de HONORI AVG, de Antonino Pio, pero ahora lleva un cetro, en lugar de la rama vista en anteriores monedas de Marco. La rama pasa al tipo de Antonino. Esto es, tal vez, un cambio de complementos entre el emperador y el príncipe. El emperador dedica un tipo a Honos en estrecha asociación con su joven colega. Marco replica con un tipo de Honos, expresando la dignidad senatorial de su señor» (MATTINGLY, 1940, LXXXVIII-LXXXIX), Honos se prolonga en todos los tipos de monedas de Marco hasta el final del gobierno de Antonino de forma continuada todos los años (9). Habría que pensar cuál es la razón de ello, que estaría más bien en la línea del carácter militar del joven príncipe en contraposición con Antonino, cuya labor sería más pacífica.

(9) STRACK, (1931, III, 144 ss.) DIO. CASS., (Hist., LXXI, 35,5) COHEN, (1880-1892, IV, 389-395). 


\section{IUVENTAS}

Es una personificación específica de Marco Aurelio como príncipe, y del hombre en edad militar, como tal se muestra en las inscripciones monetarias como Princeps Iuventutis, adoptado por Antonino cuando Marco contaba con veinte años de edad. Iuventas es la diosa de la Juventud y de la belleza en general. En el príncipe, se la invoca por ser específica de los príncipes o césares de la casa imperial. El que la posee, actúa como juez árbitro en los litigios entre los restantes miembros jóvenes de la familia imperial. También es usada como el equivalente de Hebe, la novia de Hércules, es la representación de la juventud en la mitología griega.

Iuventas es el espíritu de la juventud romana y de toda Italia, representada por Marco, la iconografía nos la presenta como un tipo de Pietas sacrificando delante de un incensario y portando pátera, es el honor que todo príncipe debe a esta diosa. Aunque no tiene ninguna relación con la posición del César en el siglo II; Antonino Pio no nombró nunca a Marco Princeps Iuventutis, por alguna razón desconocida, pero en todo caso, vinculada con la antiguas tradiciones, le otorgó a Marco el título equiparable de Sevir Turmis Equitum Romanorum, que le colocaba a todos los efectos, a la cabeza de la juventud romana. Su primera aparición en la moneda de Marco como príncipe (que por tanto está encuadrada en la de Antonino Pio), se registra en denarius de los años 140 al 144 (STRACK, 1931, III, 45ss).

Grant piensa que aunque el tipo data desde la época de Augusto, es muy sorprendente que aparezca en esta ocasión, por lo que piensa que se trata de algún aniversario especial que se conmemoraría por estos años. «En la segunda Guerra Púnica, en el día de la victoria sobre Asdrúbal en el Metauro en el año 207 d.C., el cónsul M. Livius Salinator promete hacer un templo a Iuventas (diosa de los Iuvenes, hombres en edad militar), y sería posteriormente edificado cerca del Circo Máximo. El trescientos cincuenta aniversario de este dramático evento, lo cual ha coincidido con una victoria obtenida en los años 143 al 144, es la ocasión que es conmemorada. El trescientos cincuenta aniversario bastante a menudo recibe conmemoración, el número poseía para los antiguos romanos una significación muy superior a la actual, en la medida que está a mitad de camino del séptimo centenario, y el siete era un número sagrado para los romanos» (GRANT, 1954, 223-224).

Como es de suponer el tipo se muestra también en los aes y sestertius de los años 140 al 144, donde «el príncipe representa al hombre joven entrenado en el arte de la paz y de la guerra» (MATTINGLY, 1940, IV, LXXXVII). Y se continúa con los tipos de Marco, COS II de los años 145 al 147 (MATTINGLY, 1940, IV, LXXXVII). El siguiente tipo de Iuventas, del año 148 al 149 muestra a la diosa con un trofeo militar a su lado, (MATTINGLY, 1940, IV, LXXXVIII), lo que quizás conmemore alguna victoria militar.

\section{ROMA}

Sobre el estudio de Roma existen múltiples obras dada su importancia, pero no tantas desde el punto de vista numismático, y de las representaciones de Adriano es específica la obra de C.C. Vermeule (VERMEULE, 1954, 485ss), y sobre los tipos de Roma Aeterna hay que mencionar los trabajos de A.M. Colini (COLINI, 1940, 735-736), y C. Koch (KOCH, 1940). Y sobre los tipos generales de Roma existen las obras de S.L. Cesano (CESANO, 1929, 385-400), C. Fayer (FAYER, 1976), y R. Mellor (MELLOR, 1981, 950-1.030) por citar algunas de las obras más importantes de las muchas que se pueden mencionar.

Roma es también una de las figuraciones favoritas de Adriano. Ya en el año 119 los denarius nos muestran a Roma Victrix, es similar a un tipo normal de Victoria, uno de los aspectos eternos del Estado Romano, Roma se manifiesta a la izquierda, sobre un escudo portando lanza y Victoria. Pero fue el epíteto Felix y no Victrix, con el que la honró (MATTINGLY, 1936, III, CXXX-CLXIII).

Los denarius inmediatamente posteriores, de la primera parte de los años veinte del siglo II, en esta ocasión nos muestran a Roma Victrix, ahora en pie, es tratada como una más de las deidades que protegen a Adriano (MATTINGLY, 1936, III, CXXXII). Otro denarius nos habla de la Roma Aeternae, nos muestra a la divinidad sedente sobre un escudo portando lanza y paladium, esta moneda sugiere el mismo pensamiento que la anterior, además de la celebración del cumpleaños de Roma (MATTINGLY, 1936, III, CXXXIII).

Es precisamente durante el período de los años 121 al 125 cuando Adriano antes de marcharse de 
Roma, define su época como «una nueva Edad de Oro', asociada al culto de Roma y Venus. Ello indica al pueblo romano sus deseos de bondad y favor. Antes de partir hace votos bajo la protección de las deidades romanas de la guerra y la Victoria. En los años siguientes, desde el 125 al 127, los denarius nos muestran a Roma y Virtus, deidades hermanas, que representan el poder varonil del que depende la Victoria de Roma. La figura de la diosa que porta rama, es la Roma Felix que comparte el templo de Adriano con Venus (MATTINGLY, 1936, III, CXXXIV).

Igualmente un raro quinarius de oro muestra a la diosa Roma en la postura de máximo honor, sentada, estrechando entre sus manos a la figura de Adriano (MATTINGLY, 1936, III, CLXVII). En los aes, la deidad se muestra con lanza y cornucopia como atributos; tal vez, Roma Felix es el mejor nombre para este tipo con el emblema mágico del laurel (10). Incluso en un semis de los años 125 al 128, con la única leyenda del reverso: COS, III S. C., presenta a Roma sedente a la izquierda sobre una coraza (MATTINGLY, 1936, III, CLXIX). Igualmente en los setertius comprendidos entre los años 128-132, Roma viene calificada de Victrix, lo cual coincide con los primeros momentos de la guerra de Judea (MATTINGLY, 1936, III, CXLVI).

En el año 137, después de muchos lustros de construcción se termina y se dedica el templo de Roma y Venus, a partir de este momentos, ambas deidades aparecen con frecuencia juntas en las monedas (MATTINGLY, 1936, III, CLXXXIII).

En los años 136-137 surge poco antes de la muerte de Adriano la dedicación de los bronces y como último homenaje lleva la leyenda ROMA AETERNA, en la imagen de culto de esta diosa con Victoria y lanza. Una versión de pie de esta misma aparece con el único calificativo de Roma (11).

Antonino también rinde homenaje con mucha frecuencia a Roma, ya sea la ciudad o la diosa o ambas a la vez. La primera emisión son sestertius del año 139, que llevan como leyenda ROMAE AETERNAE S.C., y en el campo un templo decástilo

(10) COHEN, (1880-1892, II, nos. 347-353); MATTINGLY y SYDENHAM, (1926, II, nº 685-C); MATTINGLY, (1936, III, CLXIX).

(11) COHEN, (1880-1892, III, $\mathrm{n}^{\circ}$ 698); MATTINGLYSYDENHAM, 1930, III, $\mathrm{n}^{\circ} 543$ ).
(12), que muy bien puede ser el templo tantas veces conmemorado por Adriano, el gran santuario inaugurado pocos años antes por su antecesor, el Templo de Roma y Venus, esta acuñación se continúa en la emisión del año 140 al 144. El gran edificio para Roma y Venus, planeado por Adriano no llegó a terminarse. Fue dedicado a Roma Aeterna y a Venus Felix. El Templo de Roma muestra a Roma sentada en el centro del tejado, y Victorias en los laterales, por el contrario, no muestra estatuas de culto de Venus. Con ello rinde no sólo homenaje a la memoria de Adriano y Sabina, sino que también es el símbolo de su aceptación por el Senado y el Pueblo de Roma. Esos mismos años, del 140 al 144, sestertius y denarius hablan de ROMA AETERNA, es el tipo creado por los acuñadores de Antonino, presenta a la diosa como la «Nueva Troya», y muestra a la diosa Roma, sosteniendo pala dium y lanza, sentada a la izquierda en un trono en el que se apoya un escudo. Strack (STRACK, 1931, 127), dice sobre esta acuñación: «Roma Aeterna es la heredera de Troya. La concepción de la "Providencia de los Dioses", como la justificación del papel imperial, el "carisma" de la Gracia Divina o unción, es bastante natural. Una idea similar fue desarrollada en el tipo de Adriano, el cual muestra un águila situada sobre él y un cetro en su garra. El cetro es el símbolo de la Divina Majestad; aquí es el rayo, el supremo emblema del poder; que es directamente comisionado para el emperador por el Señor del Cielo. Pero es innecesario ver una tentativa de hacer al emperador independiente de la suerte de su predecesor en el Senado. Es, simplemente, una explicación en términos espirituales de lo que acontece en el curso ordinario de la vida del Estado». Entre los medallones, podemos citar uno muy temprano, del año 139, que muestra a Roma sentada dándole la mano al emperador, lo cual es el viejo símbolo de la Concordia, que en este caso es la bienvenida de la ciudad al nuevo emperador (MATTINGLY, 1940, IV, XCIV). Entre las emisiones monetarias del año 145 al 147 , el principal tipo es el aureus que muestra a Roma sentada con palladium, como «segunda Troya». Existe de ese mismo período una rara variante con Roma Victrix, que muesta a la diosa sentada, sosteniendo lanza y con el co-

(12) COHEN, (1880-1892, III, nos. 621 Y 694); MATTINGLY y SYDENHAM, (1930), III, n ${ }^{\circ} 621$ A). 
do apoyado en un escudo, y que puede hacer referencia a victorias militares en Britannia, pues la acuñación se prolonga hasta el año 161 (13). Esta moneda está descrita por M: Verbeeck (VERBEECK, 1981, 144-145). También simboliza la diosa Roma que Antonino ha gobernado con prosperidad y bondad. Que es continuada por emisiones de Marco Aurelio con el mismo tipo (MATTINGLY, 1940, IV. LXXXIX y LXXII). Un segundo medallón de este mismo periodo, nos muestra a Roma saludando a Felicitas a Antonino y a Marco, que realmente tiene que expresar confianza en el joven príncipe al subir al poder (MATTINGLY, 1940, IV, XCV).

\section{ROMVLO, LOBA CAPITOLINA Y LOS GEMELOS}

Es un tipo propio de Antonino Pio, y aunque por su naturaleza de recuerdo mitológico de la fundación de Roma, habría que incluir su estudio dentro de las acuñaciones propias de este emperador; la gran cantidad de emisiones que muestran a estos legendarios personajes, obligan a abrir un capítulo aparte, que, además, se encuentra relacionado intimamente con el anterior apartado.

La primera emisión del grupo formado por la Loba Capitolina y los Gemelos data del primer año del gobierno de Antonino, lo cual viene a demostrar la gran importancia concedida, se trata de un denarius, que muestra a la Loba amamantando a los gemelos Rómulo y Remo, con la inscripción TR.P.COS.II, (MATTINGLY y SYDERHAM, 1930, III, n. ${ }^{\circ} 42$ a-S). Para Mattingly (MATTINGLY, 1940, IV, LIV), simboliza el «Renacimiento de Roma» y al igual que Strack piensa que «la renovatio de Roma e Italia es, en sí misma, una parte integral de la política de Antonino». Los sestertius de esa larga acuñación de cuatro años, comprendida entre el 140 al 144, insisten en el grupo escultórico y en lo que simboliza (14). Otros sestertius coetaneos, con la TR.POT.COS.III.S.C., muestran a Rómulo con los atributos de Marte, es decir, la lanza y el trofeo, pero a diferencia de éste no lleva el casco que le caracteriza, sería por tanto,

(13) MATTINGLY, (1940, LXIV); COHEN, (1880-1892, III, nos. 690-691); MATTINGLY y SYDENHAM, (1930, III, nos. 874 Y 892).

(14) COHEN, (1880-1892, III, nos. 917-918); MATTINGLY y SYDENHAM, (1930, III, nos. 645, 648-649).
Romulo Conditor, con el cual quiere identificarse Antonino como refundador de la Urbs. Strack (STRACK, 1931, III, 71),presenta al emperador como un «segundo Rómulo», indicando que ya Augusto quiso adoptar ese nombre para sí mismo. Es el tema del gran pasado de Roma y de la «Roma Renaciente». Los cuadrans, moneda de muy poco uso por su escaso valor y poca acuñación, muestran en el mismo período a la imagen de la Loba Capitolina y los Gemelos (MATTINGLY, 1940, IV, LXXX). No obstante, la emisión de estas iconografías son cortas en el tiempo, ya que el último tipo monetario de Rómulo tiene lugar en el año 147 (MATTINGLY, 1940, IV, LXIV).

\section{LIBERTAS}

Libertas, es a diferencia de las anteriores, una idea más difundida por todo el ámbito del Imperio, sin que por ello deje de tener relación con los símbolos ya vistos. Es así, probablemente, uno de los tipos más usados por Adriano en su propaganda numismática. Ya en los años 119-121, los sestertius portan la leyenda LIBERTAS RESTITVTA (en el exergo) PONT. MAX. COS. III. (alrededor) SC. La escena representada muestra a Adriano sentado a la izquierda en un estrado, tendiendo la mano a una mujer en pie, abajo en una escalera, con el pie izquierdo sobre una basa, en los brazos lleva a un niño, mientras que el otro permanece a su lado (15). Es el típico gesto de la Indulgentia, que posteriormente volverá en emisiones de años sucesivos. Tipo y leyenda son aparentemente discordantes y ello, según Mattingly, supone un interesante problema. Libertas Restituta naturalmente indica alguna acción para restaurar la libertad de los ciudadanos romanos.

Adriano es el legislador humanista, al igual que Trajano, promete su favor a los niños de Italia presentes ante él, este es otro buen motivo para hablar de esta alegoría de forma propagandística, utilizando el tipo más adecuado de su antecesor: el de los alimenta que vuelve a resucitar en monedas de esta época. Mattingly dice que hay que mirar el tipo como una nueva presentación de los alimenta, el emperador restaura la «Libertad» para proteger la edu-

(15) MATTINGLY y SYDENHAM, (1926, II $n^{\circ}$ 568); MATTINGLY, (1936, III, CLXIV). 
cación de los pobres huérfanos. La ternura de los romanos hacia el juego de palabras existente entre Liberi, Liber y Libertas explicaría claramente este significado, que, por otra parte, difícilmente podría ser otro. (MATTINGLY, 1936, III, CLXIV).

Los aureus y denarius en los que figura Libertas, con pileus y rama o cornucopia, es una nueva figura que aparece en el gobierno de Adriano, como ya hemos indicado más arriba. Muestra preferentemente que el emperador está dispuesto a conceder todas las libertades posibles a sus súbditos. Así, en el año 121 esta figura aparece acompañada de la leyenda LIBERTAS PVBLICA, que representa la libre respublica bajo el Optimus Princeps (16).

En aureus y denarius de los años 125 al 127, $\mathrm{Li}$ bertas está en figura y leyenda en los reversos, concretamente para esta emisión Mattingly opina (MATTINGLY, 1936, III, CXXXVI), que se trata de mirar las susceptibilidades romanas a través de hacer la propaganda por la libertad de acción de los ciudadanos, dentro de la necesaria unión del Imperio. Esto último está en íntima relación con los sestertius con la leyenda del reverso LIBERTAS RESTITVTA, emitidos en el período inmediatamente anterior. En este sentido es necesario ver la obra de A. Cothenet (COTHENET, 1972, 242-243), que expone la teoría de que el sestertius de Adriano que lleva esta leyenda está acuñado para defender un precepto del Derecho Civil, a saber, el de asegurar a la madre el derecho de recibir una parte de la herencia de los hijos difuntos. Por último, para la etapa comprendida entre los años 132 al 134, y probablemente después de la adoptio de Elio y la dedicación del templo de Roma y Venus, vuelve por última vez en el gobierno de Adriano en aes con la leyenda en el reverso: LIBERTAS PVBLICA s.c. La divinidad está de pie a la izquierda, llevando cetro y bonete. Para Mattingly (17), esta última moneda «es la Libertad que nunca debe ser perdida en Roma, ni aún en el período de transición que supone una nueva adopción, en este caso la de Elio, la herencia de la República, la Libertas, es salvaguardada por lo indicado en otras acuñaciones paralelas, es decir, la Pax y la Salus Augusti». Por último, indicar

(16) COHEN, (1880-1892, II, nos. 948-954); MATTINGLY y SYDENHAM, 1926, (II, $\mathrm{n}^{\circ} 583$ A).

(17) MATTINGLY, (1936, III, CLXXXIII); COHEN, (18801892, II, nos. 923-933); MATTINGLY y SYDENHAM, (1926, II, $\left.\mathrm{n}^{\circ} 729-\mathrm{C}\right)$. que sobre la iconografía de Libertas existe un artículo de J. Toutain (TOUTAIN, 1950, 183-187), que explica el origen de la imagen de esta deidad, y que es seguramente el más curioso de todos los orígenes conocidos de las diosas. Según Toutain, cuando la imagen de Libertas se encuentra desnuda, en algunas variantes, debe inspirarse en el retrato de una cortesana griega, basándose en el Pro Domo de Cicerón que cuenta cómo P. Clodius trae de Grecia y coloca, entre el año 57 ó 58 d.C., la imagen de la Libertas en el templo construido a esta diosa en el Palatino, de donde derivó la imagen monetaria que se muestra por primera vez en acuñaciones de Claudio.

Con Antonino, al contrario que Adriano, las emisiones con Libertas son escasas, y además con grandes diferencias cronológicas entre la primera emisión y las siguientes. No obstante, como las monedas de Antonino son Libertas no pueden ser separadas de aquellas que llevan la leyenda Liberalitas, es imprescindible consultar la obra de A.U. Stylow (STYLOW, 1972), que da cumplidas referencias de cómo el concepto de Libertas va invariablemente unido a los congiaria que el emperador reparte a sus súbditos; en la medida que se confundían los conceptos de libertad con el de prosperidad material, o simplemente de mera subsistencia, por otra parte la semejanza de ambas palabras daba un juego de términos que los romanos confundían entre sí. La primera emisión data del año 139, y porta la leyenda LIBERTAS PVBLICA (alrededor) COS. II (en el exergo). La Libertas se muestra de pie con el clásico gorro frigio, símbolo de la Libertas y el cetro de majestad, para Mattingly la mención de $L i$ bertas al comienzo de su reinado es un claro acto de anuncio de línea programática de su gobierno el cual, por supuesto, se basará en la libertad. En este caso, y como hizo Adriano al comienzo de su gobierno, debió perdonar grandes cantidades de deudas existentes o bien, hubo una remisión de tasas (MATTINGLY, 1936, III, LIII). Tras un lapso de algunos años Libertas vuelve a mostrarse el año 143, por alguna ocasión especial, aunque desconocida (MATTINGLY, 1936, III, LXXXIV). La segunda emisión con tipos de Libertas corresponde al año 153 al 154 y se prolonga hasta el 156 , en este caso, Libertas no es Pública sino simplemente $\mathrm{Li}$ bertas, que parece estar relacionado con la séptima liberalidad entregada por Antonino en el año 153 o el 154, pues como hemos indicado anteriormente, una especial generosidad en la distribución de ali- 
mentos, puede ser confundida, y de hecho esa es la impresión que quiere dar la propaganda imperial, con una inusual bondad y cuidado de los intereses del pueblo, a este respecto dice Mattingly citando a Plinio en su capítulo XXVI del Panegírico: «el incremento de la popularidad de un emperador queda reflejada en su liberalidad extendida a los niños aunque el emperador se encuentre ausente de Roma. De ahí que a menudo se haga el juego deliberado en todo el Imperio entre Liberalitas y Libertas. Libertas a su vez, comprende el juego de Liberi (niños). Esta parte de la libertad fue habilmente extendida a la familia» (MATTINGLY, 1936, III, XC).

\section{BIBLIOGRAFÍA}

Obras de carácter general

CARY, E. 1969: Dio's Roman History, London-CambridgeMassachusset.

COHEN, H. 1880-1892: Description Historique des Monnaies frappées sous l'Empire Romain, París.

GRANT, M. 1954: Roman Imperial Money. Edimburg.

MAGIE, D. 1967: Scriptores Historiae Augustae, LondonCambridge-Massachusset.

MATTINGLY, H. 1936: British Museum Catalogue of Coins of the Roman Empire, III, Nerva to Hadrian, BristolOxford.

- 1940: British Museum Catalogue of Coins of the Roman Empire, IV, Antoninus Pius to Commodus, Bristol-Oxford.

MATTINGLY, H. y SYDENHAM, E. 1926: Roman Imperial Coinage, II, Vespasian to Hadrian, London.

- 1930: Roman Imperial Coinage, III, Antoninus Pius to Commodus. London.

STRACK, P.L. 1931: Untersuchungen zur römischen Reichprägung des Zweiten Jahrhunderts. Berlín.

\section{Providentia}

ALFOLDI, M.R. 1955-1956: «Providentia Augusti», $A$ Arch. Hung. III, 245-260.

BERANGER, A. 1960: «La Prevoyence (PROVIDENTIA) imperiale et Tacite, Annales, 1, 8», Hermes, LXXXVIII, 87-106.

BLANCHET, A. 1936: «Providentia», Dictionaire des Antiquites grecques et romaines d'apres les textes et les monuments, IV $/ 1,715-716$, París.

CHARLESWORTH, M.P. 1936: «Providentia and Aeternitas», $H T h R, 107 \mathrm{ss}$.

MARTIN, J.P. 1982: «Providentia Deorum. Recherches sur certains aspects religieux du pouvoir imperial romain, Roma.

SCOTT, R.T. 1982 «Providentia Aug», Hist., XXXI, 436-459.

WISSOWA, G. 1912: Religion und Kultus der Römer, 259, Mónaco.
Un aspecto especial de la providentia: la adoptio

ALTHEIM, F. 1955: Historia de Roma, Madrid.

BERANGER, J. 1939: «L'heredité du Principat. Note sur la transmision du pouvoir imperial aux deux premiers siecles», $R E L, 171-187$.

BRASSLOFF, S. 1914: «Die Rechtstrage bei der adoption Hadrianus», Hist., XIV, 590ss.

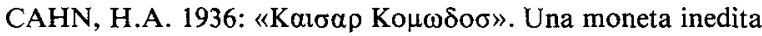
di Adriano e Elio coniata a Stratonikeia-Adrianopolis», $\mathrm{Nu}$ mismatica II, 57-58.

CAMUZZI, G. 1900: «Intorno al adoptio di Adriano Imperatore», $R I, 157 \mathrm{ss}$.

CARCOPINO, J. 1949: «L'heredité dynastique chez les Antonins, $R E A$, LI, 262ss.

- 1965: «Encore la succesion d'Hadrien», REA, LXVII, 67-69.

DEN BOER, W. 1955: «Trajan's deification and Hadrian's sucession», Studies in graeco-romans history, 227-286.

FLERES, G. 1951-1964: «Plotina e la Succesione di Adriano», Atti Accademia Peloritana (Messina), XLVIII, 199-205.

GEER, R. M. 1936: «Second thoughts on the imperial succesion from Nerva to Commodus", TAPhA, 47-54.

GRENADE, P. 1950: «Le réglament succesorial d'Hadrien», REA, LII, 258ss.

MAZZARINO, S. 1980: L'Impero Romano. Bari.

NESSELHAUF, H. 1955: «Die adoption des römischen Kaisers», Hermes, LXXXIII, 247ss.

PFLAUM, H. G. 1963-1964: «Le reglament succesorial d'Hadrien», Historia Augusta Colloquium, 95-121.

PREVOST, M. H. 1949: Les adoptions politiques a Rome sous la République et le principat, París.

VALERA, G. 1973: «Una tradizione ceionia nella Historia Augusta», RAAN, XLVIII, 1973, págs. 135-194.

\section{Honos}

COOK, A. B. 1925: «Zeus», Cambridge Ancient History, II, I, 96 ss. Cambridge.

GRANT, M. 1954. Roman Imperial Money, 150-151 y 261, Edimburg.

PLATNER, S. T. y ASHBY, T. H. 1929: A Topographical Dictionary of Ancient Rome, 258-260, Edimburg-London.

SANTER, A. 1983: $P W$, VIII, 2.292-2.294.

SCICHILONE, G. 1958-1966: «Honos», Enciclopedia dell'Arte Antica Classica e Orientale, IV, 54 y 55. Roma.

WISSOWA y DREXLER, 1884-1937: Ausführliches Lexiconder griechischen und römischen Mythologie, I, 2, 2.7072.709. Berlín.

WISSOWA, G. 1912: «Honos», Religion und Kultus der Römer, III, 1, 248. Mónaco.

Ivventas

GRANT, M. 1954: Roman Imperial Money, 109, 151, 244 ss. Edimburg.

KÖLER, W. 1958-1966: «Iuventas», Enciclopedia dell'Arte Antica Classica e Orientale, IV, 279-280. Roma.

STRACK, P.L. 1931: Untersuchungen zur römischen Reichprägung des Zweiten Jahrhunderts, III, 45 y 109. Berlín. 
WISSOWA, G. 1912: Religion und Kultus der römer, II, 135 ss. Mónaco.

\section{Roma y Rómulo}

AMELUNG, V. 1903: Die Sculturen des Vaticanischen Museums, I, Berlín.

BELLONI, G.G. 1960: Le monete romane dell'etá reppublicana. Milano.

BLANCHET, A. 1940: Mercure et Rome sur un medaillon de bronze estampé. París.

BISI, A. 1958-1966: «Personificazioni di Roma Antica», Enciclopedia dell'Arte Antica Classica e Orientale, VI, 889-901. Roma.

CESANO, S.L. 1929: La figura di Roma sulle monete romane. Roma.

COLINI, A.M. 1940: «Rom Aeterna», Capitolium, XV, 735-736.

CAGIANO DE AZEVEDO, M. 1954: «La Dea Barberini», RIA, III, $108-146$.

CALZA, G. 1926-1927: «La figurazione di Roma nell'Arte Antica», Dedalo, VII, 663-688.

CROUS, J.W. 1937: «Roma auf Waffen», Corolla Ludwig Curtius, Stoccarda, 217-224, tav. LXXXIl.

CUMONT, F. 1896: «L'eternité des empereurs romains», $R H L R$, 449.

DI SALVO, S. 1970: "Il mito di Roma Aeterna", Labeo, XVI, 95-98.

FAYER, C. 1976: Il culto della Dea Roma, Roma.

HABERLIN, E.J. 1906: «Der Roma -Typus auf den Münzen der römischen Republik», Corolla numismatica B. Head, tav. VI, 9, Oxford.

HERON DE VILLEFOSSE, A. 1989: «Le trésor de Boscorealè, Mon. Piot, V. Parigi.

KLUGNIANN, A. 1879: L'effigie di Roma nei tipo monetarii piu antichi, Berlín.

KNOCHE, U. 1949: Uber die Dea Roma. Ein Sinnbild römischen Selbstauffasung, Köln.

KNOCHE, U. 1953: «Die Augusteische Ausprägung der Dea Roma», Gymnasium, LIX, 324-349.

KOCH, C. 1949: Roma Aeterna, Roma.

LUCAS, H. 1901: «Roma auf Säulenkapitäl in der CaracallaThermen», MDAI (R), XVI, 248ss.

MAGI, F. 1945: I relievi Flavi del Palazzo della Cancelleria, Roma.
MAYNIAL, E. 1877-1919: «Roma», Dictionnaire des Antiquités grecques et romaines d'apres les textes et les monuments, IV.2, 875-878, París.

MELLOR, R. 1981: «The Goddess Roma», $A N R W$, I1, 17.2, 950-1030.

PARISOTTI, A. 1988: «Evoluzione del tipo di Roma nelle rappresentanze figurate dell' antichitá classica», Archivio di storia patria, XI, 59-148, Roma.

RICHTER, F. 1909-1915: Ausführliches Lexiconder griechischen und römischen Mythologie, IV, 130-164.

SCHROEDER, A.J. 1982; «Virgilio y la sacralidad de un mito», AHAM, XXIII, 295-318.

STRONG, E. 1907: Roman Sculture from Augustus to Constantine, London-New York.

VERBEECK, M. 1981: «Romeneise munt te Leuven», $R B N$, CXXVII, 144-145.

VERMULE, C. C. 1954; «A Hadrianic representation of Roma on coins, gems, and bronze disc», NCirc, 485 ss.

- 1960: The Goddess Rome in the Art of the Roman Empire, Cambridge-Massachusetts.

\section{Libertas}

BLANCHET, A. 1877-1919: "Libertas», Dictionnaire des Antiquités grecques et romaines d'apres les textes et les monuments, III/2, 1199, París.

COTHENET, A. 1972: «Libertas Restituta. Essai d'Interpretation», BSFN, XXVII, 242-243.

GAICHAS, L. E. 1972: Concepts of Libertas in Sallust. The Ohio State University.

JENS, W. 1956: Libertas bei Tacitus, Bonn.

KLOESEL, H. 1935: Libertas, Breslau.

KÖHLER, W. 1958-1966: «Libertas», Enciclopedia dell'Arte Antica Classica e Orientale, IV, 614-615, Roma.

STYLOW, A.U. 1972: Libertas und Liberalitas. Untersuchungen zur innepolitischen Propaganda der Römer, München.

TERLINDEN, CH. 1961: «Les Dioscures dans la litterature et la numismatique romaines», BIBR, XXXIII, 89-99.

TOUTAIN, J. 1950: «Sur une image romaine de la Liberté», BCTH, 183-187.

WIRSZUBSKI, CH. 1950: Libertas as a political Idea at Rome during the Late Republic and Early Empire, Cambridge. 